\title{
Evaluation of Protective Effect of Methyl Sulfonyl Methane on Colon Ulcer Induced by Alendronate
}

\author{
Safaa William Mdawar ${ }^{1}$, Shaza Anwar Al Laham ${ }^{1, *}$ and Ahmad Izzat Al-Manadili² \\ ${ }^{1}$ Pharmacology \& Toxicology Department, Faculty of Pharmacy, Damascus University, Damascus, Syria \\ ${ }^{2}$ Oral Histopathology Department, Faculty of Dentistry, Damascus University, Damascus, Syria
}

\begin{abstract}
Background: Bisphosphonates represent a new class of drugs that have shown very promising therapeutic efficacy in the treatment of a number of diseases associated with abnormally accelerated bone resorption including osteoporosis, Paget's disease, and hypercalcemia of malignancy. As an increasing number of these drugs become available for clinical use in the treatment of the millions of individuals with these skeletal diseases, evidence is becoming available that their chronic usage may be associated with a number of gastrointestinal side effects, such as diarrhea, abdominal pain and inflammation, and erosions and ulceration of the upper gastrointestinal tract. Methyl sulfonyl methane (MSM) which is used for osteoarthritis was used to prevent bisphosphonates ulceration. Our objective was to study the preventive effect of MSM against colon ulcer induced by Alendronate (ALN) in rats. The experiments had been done on 8 white wistar rats for each group. The gastric ulcer has been induced by administration of Alendronate $(20 \mathrm{mg} / \mathrm{kg} /$ day) by gavage for 4 days. MSM $(400 \mathrm{mg} / \mathrm{kg} / \mathrm{day})$ has been given for the protective group for 4 days before administration of Alendronate. The ulcers in rats' colon were examined histologically and microscopically. The results showed that administration of MSM before Alendronate inducing ulcer led to a reduction in ulceration and showed significant difference comparing with morbidity group.
\end{abstract}

Conclusion: MSM (400 mg/kg/day) has protective effect of colon ulcer induced by alendronate.

Keywords: Gastrointestinal, Colon ulcer, Protective, Alendronate, Methyl sulfonyl methane.

\section{INTRODUCTION}

Causes of colonic ulcers include inflammatory bowel disease, infection, medication-induced ulceration, vasculitis, fecal stasis, rectal prolapse, renal failure, and malignancy. Colonic injury and inflammation has been attributed to a number of orally and rectally administered substances. These agents may cause colonic injury through a number of mechanisms, including direct mucosal injury and ischemic injury. The injury could be ulcerative or non-ulcerative [1].

Bisphosphonates are a family of chemically related zwitterionic molecules that are used clinically to retard bone resorption in individuals with osteoporosis and associated skeletal diseases. Inflammation and ulceration of the upper gastrointestinal tract by a mechanism that relates to a topical irritant action is associated with the consumption of some bisphosphonates [2].

MSM is a naturally occurring organosulfur molecule and a putative methyl donor. MSM is the first oxidized metabolite of dimethylsulfoxide (DMSO). In the troposphere, DMSO is a byproduct of phytoplankton and algae decay. In commercial production, MSM is synthesized by reacting DMSO and hydrogen peroxide,

*Address of correspondence to this author at the Pharmacology \& Toxicology Department, Faculty of Pharmacy, Damascus University, Damascus, Syria; Tel: 00963933375655; E-mail: lahamshaza@gmail.com

ISSN: 2223-3806 / E-ISSN: 1927-5951/17 which yields MSM and water. In the body, approximately $15 \%$ of orally ingested DMSO is metabolized into MSM [3]. Health claims associated with MSM include relief of pain, inflammation, arthritis, allergies, certain parasitic infections and asthma. It is also used to nourish skin, hair and fingernails, due to its sulfur concentration, which contributes to cystine, a sulfur amino acid that is required for the production of keratin [4].

\section{MATERIALS AND METHODS}

\subsection{Animals}

Twenty-four male albino rats of the Wistar strain weighing between 180-250 $\mathrm{g}$ were used for this study. The animals were separated randomly into six cages of four rats each where they were kept for four weeks before the start of the experiment. The animals were housed under standard conditions of temperature (23 \pm $\left.2^{\circ} \mathrm{C}\right)$, humidity $(55 \pm 15 \%)$ and 12 -hour light $(7.00 \mathrm{am}$ $7.00 \mathrm{pm})$. The cages were constantly cleaned in order to prevent the animals from contracting disease. They were fed with standard commercial rat pellets and allowed water and libitum.

\subsection{EXPERIMENTAL DESIGN}

\subsubsection{Grouping}

The animals were divided into three groups of eight rats each.

(c) 2017 Lifescience Global 
Group One (group A) (NORMAL): Animals were treated with normal saline for four days, then they were treated with normal saline $+\mathrm{NaOH}$ for four days. They were called the control group.

Group Two (group B) (Alendronate ALN): Animals were treated with normal saline for four days before Alendronate administration (20 $\mathrm{mg} / \mathrm{kg} / \mathrm{day})$ for four days.

Group Three (group C) (MSM): Animals were treated with $400 \mathrm{mg} / \mathrm{kg}$ of Methylsulfonylmethane for four days before Alendronate administration (20 $\mathrm{mg} / \mathrm{kg} / \mathrm{day}$ ) for four days.

\subsubsection{Ulcer Induction}

Animals were singly housed and fasted for $18 \mathrm{~h}$ in wide mesh bottom cages, allowed free access to water except for the last hour before the last dose of the medication. Alendronate was administrated by gavage (20 $\mathrm{mg} / \mathrm{kg} / \mathrm{day}$ ) for four days [5]. Then they were euthanized and killed under deep ether anesthesia.

\subsubsection{Operative Procedure}

Immediately after the animals were killed, the entire colon starting from caecum was excised, longitudinally split. Washed with ice-cold saline to remove fecal residues.

\subsection{Macroscopic Damage Score}

The colonic samples were scored macroscopically according to the following grading system: $0=$ noinflammation; 1 =swelling or redness; $2=$ swelling and redness; $3=$ one or two ulcers; $4=$ more than two ulcers or one large ulcer; $5=$ mild necrosis; $6=$ severe necrosis [6].

\subsection{Histopathological Study}

After formalin fixation (10\% during 24 hours), each excised sample block was processed for histological evaluation. The sample block was first dehydrated by immersion in progressively increasing concentrations of ethanol and then xylene. Following this, the dehydrated tissue was immersed in melted paraffin at $60{ }^{\circ} \mathrm{C}$ for $3 \mathrm{~h}$ before being embedded in a paraffin block. 5-micron thick sections were cut by using an 82-spence microtome. The sections were then deparaffinized by treatment with xylene, ethanol and water. Tissues were stained with hematoxylin and eosin (H\&E) and then left in the fume cupboard overnight [7].

All groups were histopathologically assessed by using following score [8]:
$0=$ normal; $1=$ mild mixed infiltration in the lamina propria; $2=$ focal superficial ulceration of the mucosa only; $3=$ deep ulceration penetrating colonic wall through mucosa till muscularis mucosa and severe inflammation; 4=necrosis through large bowel wall.

\subsection{Statistical Analysis}

All obtained values were expressed as mean \pm standard deviation (SD). Data analyses were achieved using prism (Version 5) statistical package. Lesion score and histological score (non-parametric values) analyzed using the Kruskal-Wallis nonparametric analysis of variance with mann-whitney comparison test. $P$ values less than 0.05 were considered statistically significant.

\section{RESULTS}

\subsection{Macroscopic Study}

The macroscopic findings of opened colon showed healthy mucosa in most samples with no swelling or redness, and some tiny pinpoint hemorrhages in Control group (group A). After administration of Alendronate the colon mucosa appeared

Table 1: Effect of MSM on Alendronate-Induced (ALN; 20 mg/kg, p.o.) Colon Injury Macroscopically

\begin{tabular}{|c|c|c|c|}
\hline Group & Control & Alendronate & MSM \\
\hline \hline Grade & $1 \pm 1.852$ & $4 \pm 1.069$ & $2.625 \pm 0.9161$ \\
\hline
\end{tabular}

Values are expressed as mean \pm SD (Standard Deviation), one-way ANOVA followed by mann-whitney's test as compared to control. AIN.: alendronate.

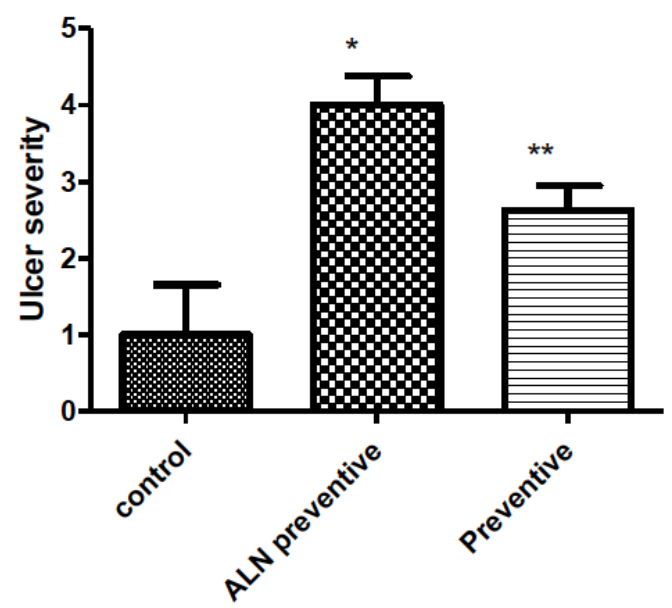

Figure 1: Comparing ulcer severity macroscopically between groups.

*significant difference between group $B$ and group $A$.

**significant difference between group $C$ and group $B$. 


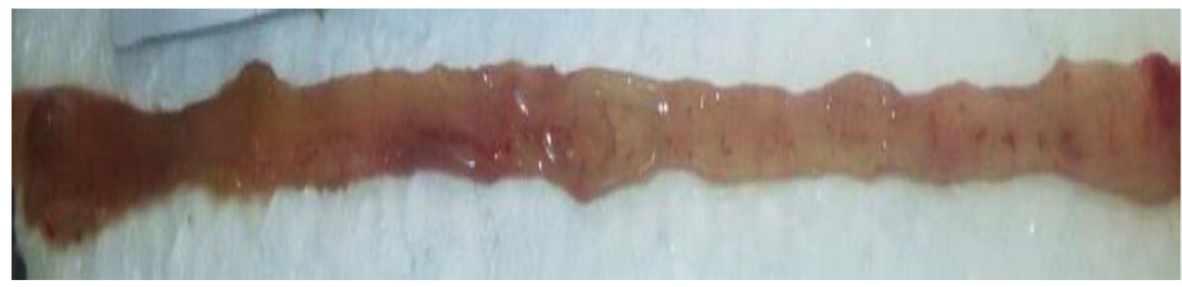

Figure 2: Normal colon- Control group- grade 0.

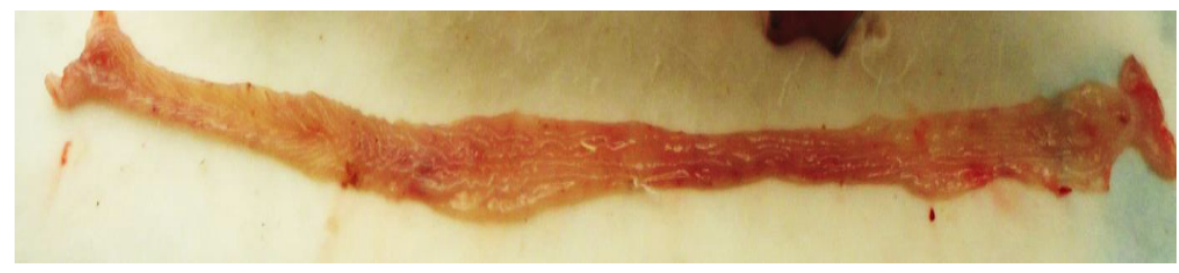

Figure 3: swelling and redness - MSM group- grade 2.

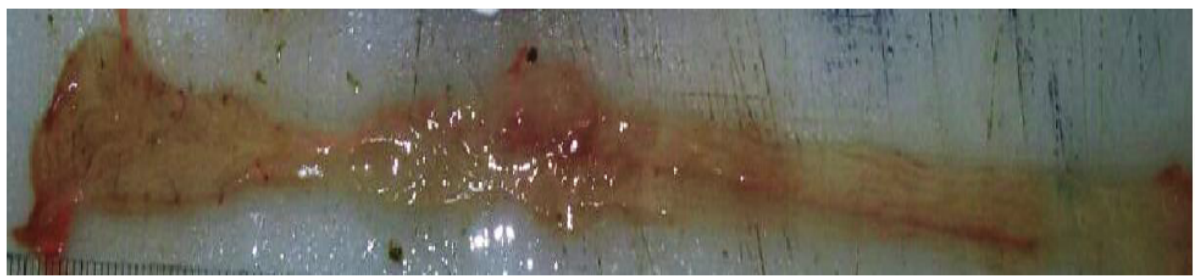

Figure 4: Large erosions with hemorrhage- ALN group- grade 4.

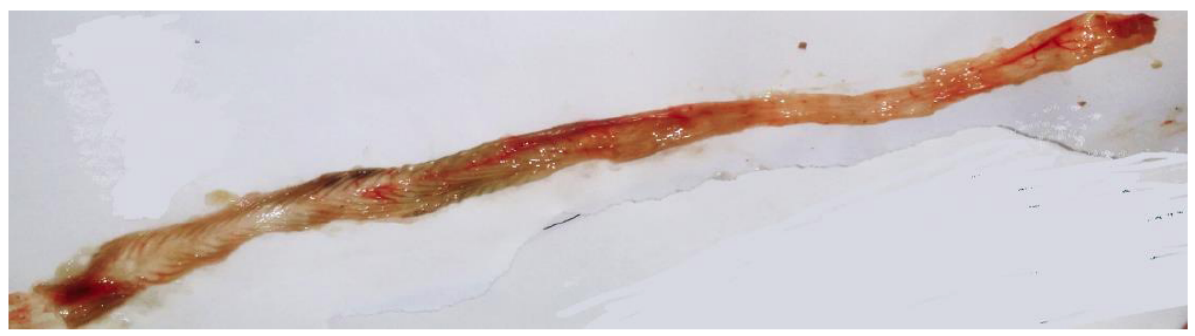

Figure 5: Large erosions with necrosis- ALN group- grade 5.

macroscopically ulcerated and hemorrhagic. It also showed necrosis in some samples in Alendronate group (group B) compared to control group $(p<0.05)$. Administration of MSM inhibited Alendronate-induced ulcer formation, so that the macroscopic findings showed less ulcerated regions and pinpoint hemorrhages. Thus there is significant difference when comparing protective group which took MSM (group C) with Alendronate group (group B); $p<0.05$.

Table 2: Effect of MSM on Alendronate-Induced (ALN; 20 mg/kg, p.o.) Colon Injury Histologically

\begin{tabular}{|c|c|c|c|}
\hline Group & Control & Alendronate & MSM \\
\hline \hline Grade & $1.250 \pm 0.8864$ & $2.750 \pm 0.4629$ & $1.5 \pm 0.5345$ \\
\hline
\end{tabular}

Values are expressed as mean $\pm S D$ (Standard deviation), one-way ANOVA followed by mann-whitney's test as compared to control. AIN.: alendronate.

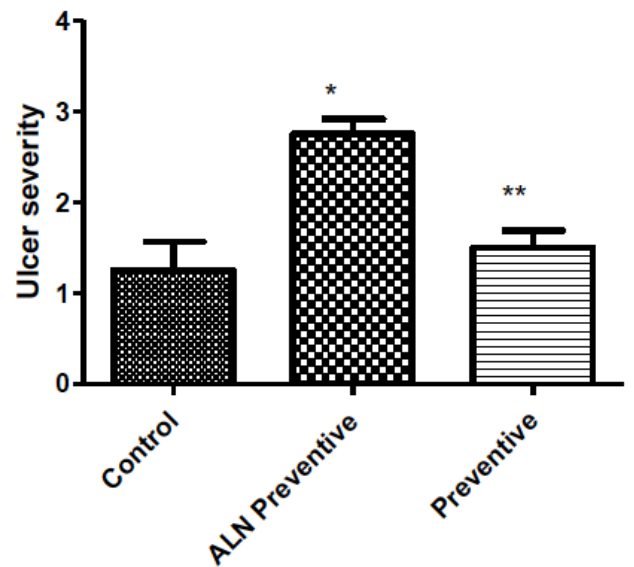

Figure 6: Comparing ulcer severity histologically between groups.

*significant difference between group B and group A.

** significant difference between group $C$ and group $B$. 


\subsection{Microscopic Study}

The colon epithelium of control group contained swelling vessels. Besides mild mixed infiltration in the lamina propria in most samples. The Alendronate group was totally ulcerated. Most samples showed deep ulceration penetrating colonic wall through mucosa till muscularis mucosa and severe inflammation. There was significant difference when comparing group $B$ with control group $(p<0.05)$. There was no ulceration in MSM group, except for some focal superficial ulceration of the mucosa only in some samples, and there was significant difference when comparing with group $B(p<0.05)$.

\section{DISCUSSION}

Esophageal injury caused by alendronate is well characterized, but there are no reports of injury to the colon except one clinical study. Biopsy specimens from

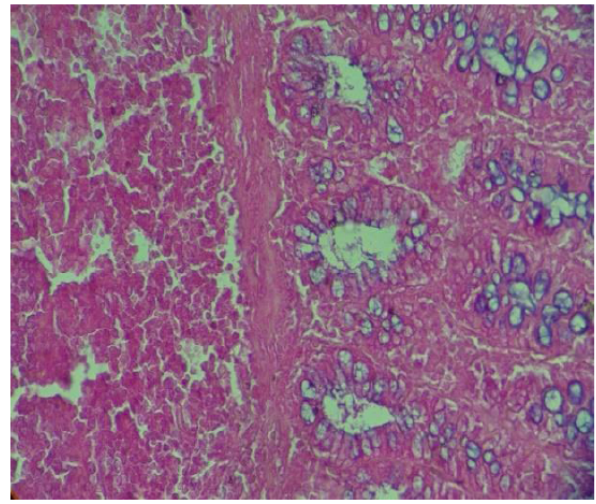

$\mathrm{x} 40$

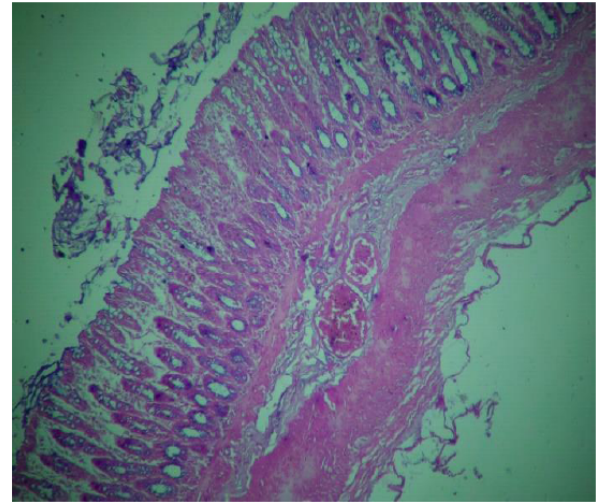

$\mathrm{x} 10$

Figure 7: Microscopic sketch for colon in different magnification- control group- grade1.

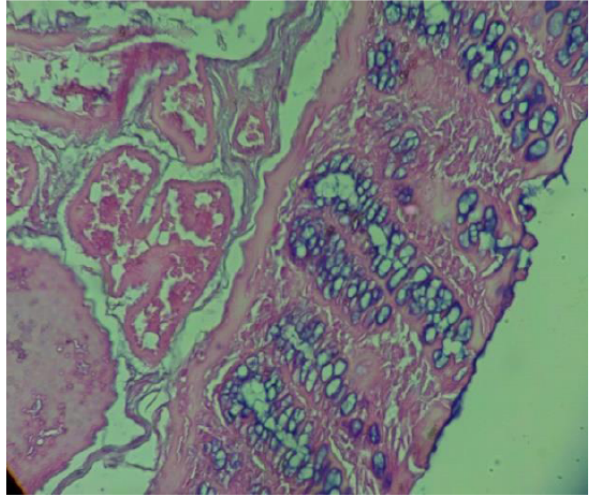

x40

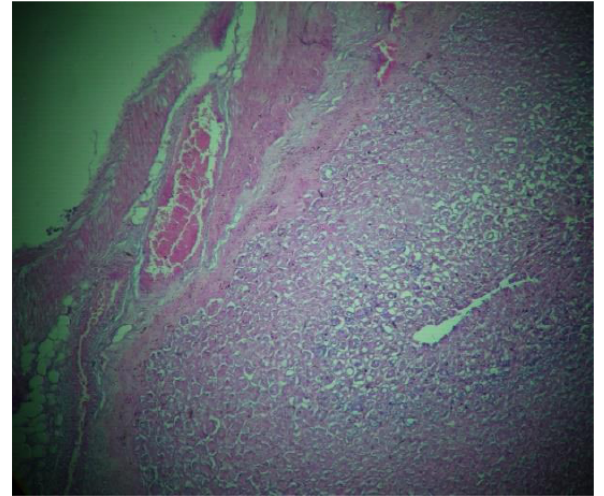

$\mathrm{x} 10$

Figure 8: Microscopic sketch for colon in different magnification- MSM group- grade 1.
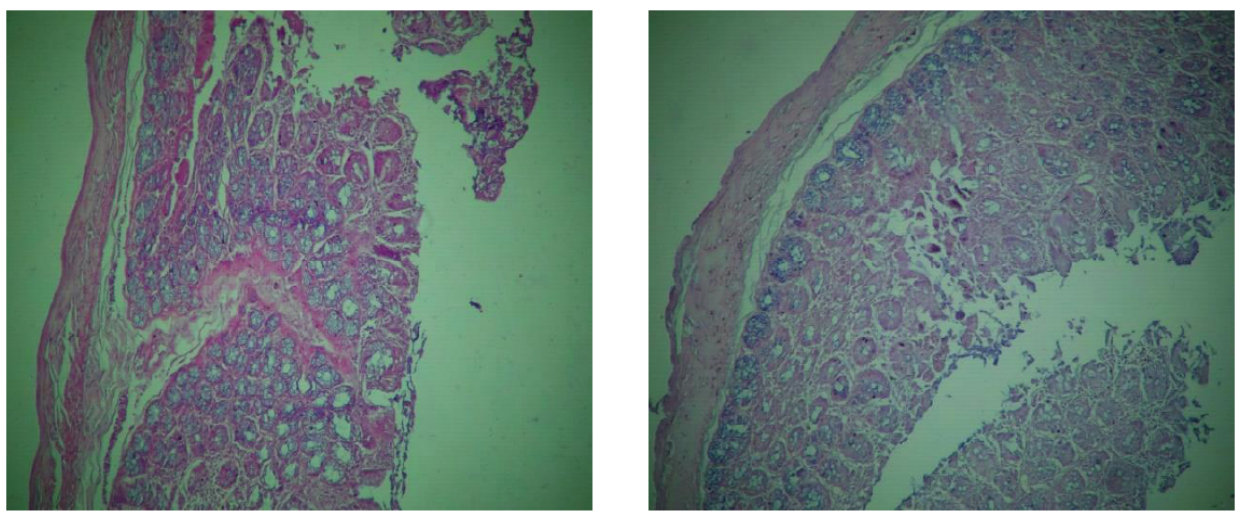

Figure 9: Microscopic sketch for colon in different magnification- ALN group- grade 2. 


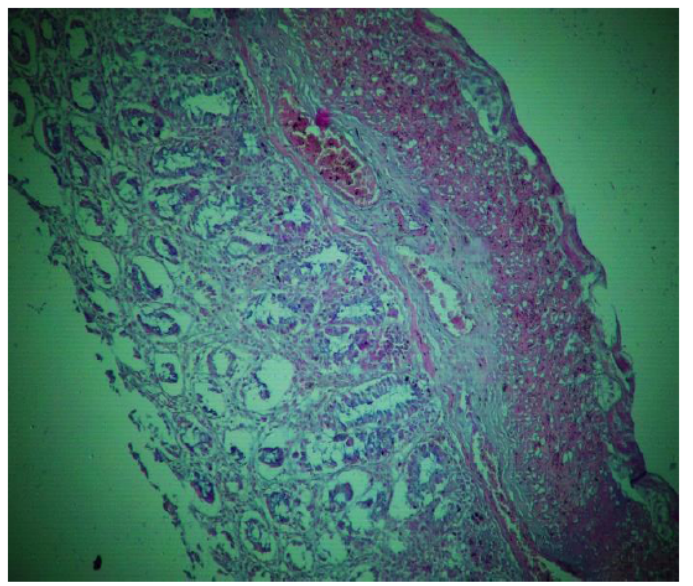

Figure 10: Microscopic sketch for colon (x10 magnification)ALN group- grade 3 .

the lesion revealed granulation tissue with reactiveappearing overlying epithelium [9]. The study was on two patients who had been initiated treatment with alendronate within 6 months of detection of the colonic ulcer. Other known causes of colon ulcers were excluded by histopathologic examination and culture of biopsy specimens. After discontinuation of alendronate, there was complete ulcer healing in both patients [9].

In our study, the ulcer was induced by administration of Alendronate $20 \mathrm{mg} / \mathrm{kg} /$ day -dissolved it in saline- for four days. This dose cause colon injury range from hemorrhage to large ulcers and mild necrosis. The histological study showed severe injury in the epithelium and deep ulceration penetrating colonic wall through mucosa till muscularis mucosa. It also showed infiltration of neutrophils and macrophages to the mucosa. This represent an early characteristic of inflammation. The neutrophils, once in contact with the gut mucosa and the lipopolysaccharides, actively produce pro- inflammatory cytokines, interleukin-1 (IL-1), tumour necrosis factor alpha (TNF- $\alpha$ ). It was observed that cytokines stimulate monocytes, macrophages and lymphocytes, responsible for maintaining inflammation [10]

The mechanism of alendronate-induced mucosal toxicity has not been completely elucidated. Experimental evidence suggests that bisphosphonates directly irritate the $\mathrm{GI}$ mucosa and may compromise the hydrophobic mucosal phospholipid layer [9].

The macroscopically observations showed different results from Amirshahrokhi et al. and Al-bitar et al. who induced ulcerative colitis in rats by using acetic acid. These showed ulcerated mucosa, hemorrhagic, oedematous and necrotic compared to normal control group, while this study didn't show the same necrotic.
Otherwise, the microscopically observations showed inconsiderable difference between those studies and the recent study. They all showed deep ulceration of muscularis mucosa, severe inflammation and necrosis through large bowel wall. In the other hand, Amirshahrokhi et al. and Al-bitar et al. results showed infiltration of small round cells and polymorphonuclear leukocytes to lamina properia $[6,7]$, that is not shown in this study. Thus, this colon ulceration is not totally the same model of ulcerative colitis induced by the acetic acid.

Nagar described Isolated ulcers of the large intestine as ulcers that are not associated with an underlying colitis and may be an incidental finding on screening colonoscopy or present with abdominal pain, hematochezia, chronic gastrointestinal bleeding, and rarely, perforation. He suggested that the common cause of isolated colonic ulcers is the use of nonsteroidal anti-inflammatory drugs (NSAIDs) [11]. This kind of ulceration is also not the same of the ulceration in this study. Besides, there was no gastrointestinal and hemorrhagic symptoms in the experimental rats.

This kind of the inflammation may be the same inflammation induced by the hapten 2,4,6trinitrobenzenesulfonic acid in previous study. That chronic inflammation of the intestine may occur as a consequence of increased permeability of the mucosa to a luminal antigen which cannot be readily cleared by the immune system [12].

Neutrophils play a crucial role in the development and full manifestation of gastrointestinal inflammation, also neutrophil infiltration into inflamed tissue plays a crucial role in the destruction of foreign antigens and in the breakdown and remodeling of injured tissue [13]. Infiltration of neutrophils result in the production of cytotoxic reactive oxygen species (ROS) that are destructive on intestinal cell macromolecules, ultimately leading to mucosal disruption and ulceration [14]. Activation of intestinal immune system is associated with excessive generation of inflammatory cytokines such as tumor necrosis factor- a (TNF- $\alpha$ ) which amplifies the inflammatory cascade by triggering the generation of other pro-inflammatory cytokines and enhancing the recruitment of macrophages and neutrophils [15].

Methylsulfonylmethane (MSM), also known as dimethyl sulfone, is an organic sulfur compound mainly present in foods such as fruits and vegetables, and in beverages as well [16]. Our study demonstrated the 
ability of MSM in protection of Alendronate induced ulceration in rats. Similar results were observed by Amirshahrokhi et al. and Al-bitar et al. who used other model of ulcerative colitis to test the anti-inflammatory and antioxidant potential of MSM $[6,7]$.

Bohlooli et al. study suggested that MSM pretreatment could alleviate hepatic injury induced by acetaminophen intoxication, this may be through its sulfur donating and antioxidant effects. Thus, it is possible to suggest that MSM as a pretreatment agent has a potential to be investigated as an agent in limiting the drug-induced oxidative damage [17].

Many studies have demonstrated that MSM inhibits the translocation of the p65 subunit of nuclear factor (NF)-kß to the nucleus, thus minimizing downstream events associated with local and systemic inflammation. Indeed, MSM may minimize the expression of pro-inflammatory cytokines. MSM has been reported to increase antioxidant defense (glutathione), as well as decrease the actual production of ROS. As with pro-inflammatory biomarkers, MSM resulted in a lowering of multiple oxidative stress biomarkers. Other studies showed that sulfur is an important constituent of amino acid(s), which contribute substantially to the maintenance and integrity of cellular systems by influencing cellular redox state and cellular capacity to detoxify toxic compounds, free radicals and ROS [7].

\section{CONCLUSION}

Methylsulfonylmethane (MSM), which have antioxidant and anti-inflammatory effect, can give protective effect of colon ulcer induced by alendronate when it is administrated in a dose of $400 \mathrm{mg} / \mathrm{kg} /$ day.

\section{REFERENCES}

[1] Yamada T, Alpers DH, Kalloo AN, Kaplowitz N, Owyang C, Powell DW. Textbook of gastroenterology, 5th ed., Chichester, 2009.

[2] Lichtenberger LM, Romero JJ, Gibson GW, Blank MA. Effect of bisphosphonates on surface hydrophobicity and phosphatidylcholine concentration of rodent gastric mucosa. Dig Dis Sci 2000; 45: 1792-1801. https://doi.org/10.1023/A:1005574009856

[3] Kim LS, Axelrod LJ, Howard P, Buratovich N, Waters RF. Efficacy of methylsulfonylmethane (MSM) in osteoarthritis pain of the knee: A pilot clinical trial. Osteoarthr Cartil 2006; 14: $286-294$

https://doi.org/10.1016/j.joca.2005.10.003
[4] Horváth KS.A, Noker PE, Somfai-Relle S, Glávits R, Financsek I. Toxicity of methylsulfonylmethane in rats. Food Chem Toxicol 2002; 40: 1459-1462.

[5] Şener G, Kapucu C, Cetinel S, Cikler E, Ayanoğlu-Dülger G Gastroprotective effect of leukotriene receptor blocker montelukast in alendronat-induced lesions of the rat gastric mucosa. Prostaglandins Leukot Essent Fat Acids 2005; 72 : $1-11$

https://doi.org/10.1016/j.plefa.2004.04.005

[6] Amirshahrokhi K, Bohlooli S, Chinifroush MM. The effect of methylsulfonylmethane on the experimental colitis in the rat. Toxicol Appl Pharmacol 2011; 253: 197-202. https://doi.org/10.1016/j.taap.2011.03.017

[7] Al Bitar V, Laham S. Mtyhylsulfonylmethan and green tea extract reduce oxidative stress and inflammation in an ulcerative colitis. Asian J Pharm Clin Res 2013; 6: 153-158.

[8] Medhi B, Prakasha A, Avti K, Saikia UN, Pandhia P, Khanduja KL. Effect of manuka honey and sulfasalazine in combination to promote antioxidant defense system in experimentally induced ulcerative colitis model in rats. Indian J Exp Biol 2008; 46: 583-590.

\section{https://doi.org/10.1177/073346488700600302}

[9] Sawhney MS, Nelson DB. Alendronate-induced colon ulcers: case report of a new clinical entity. Am Soc Gastrointest Endosc 2004; 60: 14-16.

[10] D'Ovidio V, Aratari A, Viscido A, Marcheggiano A, Papi C Capurso L, Caprilli R. Mucosal features and granulocytemonocyte-apheresis in steroid-dependent/refractory ulcerative colitis. Dig Liver Dis 2006; 38: 389-394. https://doi.org/10.1016/j.dld.2005.12.005

[11] Nagar AB. Isolated colonic ulcers: Diagnosis and management. Curr Gastroenterol Rep 2007; 9: 422-428. https://doi.org/10.1007/s11894-007-0053-9

[12] Morris G, Beck P, Herridge M, Depew W, Szewczuk M Wallace J. Hapten-induced model of chronic inflammation and ulceration in the rat colon. Gastroenterology 1989; 96 : 795-803. https://doi.org/S0016508589001058[pii]

[13] Di Paola R, Mazzon E, Patel NSA, Genovese T, Muià C Thiemermann C, De Sarro A, Cuzzocrea S. Beneficial effects of GW274150 treatment on the development of experimental colitis induced by dinitrobenzene sulfonic acid. Eur J Pharmacol 2005; 507: 281-289. https://doi.org/doi:10.1016/j.ejphar.2004.11.041

[14] Nagib MM, Tadros MG, Elsayed MI, Khalifa AE. Antiinflammatory and anti-oxidant activities of olmesartan medoxomil ameliorate experimental colitis in rats. Toxicol Appl Pharmacol 2013; 271: 106-113. https://doi.org/10.1016/j.taap.2013.04.026

[15] S.-M. F, D.-L. A, Role of cytokines in inflammatory bowe disease. World J Gastroenterol 2008; 14: 4280-4288.

[16] N. SP, Darvin P, Yoo YB, et al. The combination of methylsulfonylmethane and tamoxifen inhibits the Jak2/STAT5b pathway and synergistically inhibits tumor growth and metastasis in ER-positive breast cancer xenografts. BMC Cancer 2015; 15: 474. https://doi.org/10.1186/s12885-015-1445-0

[17] Bohlooli S, Mohammadi S, Amirshahrokhi K, et al. Effect of methylsulfonylmethane pretreatment on acetaminophen induced hepatotoxicity in rats. Iran J Basic Med Sci 2013; 16: 896-900. 